\title{
A correlation between Balmer and soft X-ray emission from stellar and solar flares
}

\author{
C.J. Butler ${ }^{1}$, M. Rodono ${ }^{2}$ and B.H. Foing ${ }^{3}$
}

1 Armayh Obscruatory, Colleye Hill, Amuayh, BTö1 9DG, N. Ireland.

2 Instituto di Astronomia, Universita di Catania and Osservatorio Astrofisico, I-95125, Catania, Italy.

'Centre National de la Recherche Scientifique, Institut d'Astrophysique Syatiale, B.P. 10, 91371 Verricrs le: Buissun, France.

Summary: Sinultaneous optical spectroscopy and $X$-ray monitoring of stellar and solar flares shows that a well-idefined linear correlation exists between the integrated $H \gamma$ and soft X-ray flux that extends over four orders of magnitude. The existence of this relationship implies a direct proportionality between the enission from the cooler and denser regions $\left(T \approx 10^{4} K\right)$ responsible for Balmer lines and the enission from the hot plasma $\left(T \approx 10^{7} K\right)$ responsible for soft $X$-rays. The conseguences are considered for (a) several models which have been proposed for solar flares, and (b) the suggestion that the Bahner cunission results from irradiation by suft $X$-rays.

\section{Introduction}

It is inportant, when trying to unravel the complex phenomena involved in stellar flares, to be able to assess where the enission in the different spectral regions arises and to what other parts of the spectrum it is related. For instance, the currently accepted picture of the evolution of a flare involves two basic phatses; first the inpulsive phase seen in hard X-rays and the optical continum, and secondly a gradual, or thermal, phase involving a siow decline in soft $X$-rays and emission lines. The first plase is usually atssociated with heating by very energetic particles (electrons or protoms) and the second is associated with a hut plasmat couling by radlation and expansion. The radiation enitted by the two phases may, or may not, correlate, depending un how closely they are linked energetically. To investigate which parts of the emission from flares correlate with one another recuires observations of different spectral regions with many different instruments simultameously. This paper concerns a search for a correlation between Balmer flux and soft $\mathrm{X}$-raty flux involving several different satellites and ground-based telescopes.

Whilst II c has traditionally been the favourite Balner line for solar work, the inportance of the near ultraviolet continumu and the greater mumber of strong lines atvailable there, hats lead to a preference by stellar astronomers for the 3500-4500 A wavelength region. The strongest Balner line in this wavelength region is $I I \gamma$ which also has the advantige that it is less optically thick than $H$ r. Where $H \gamma$ has not been the preferred option and $H a$ observations have been obtained (e.g. in the case of solar llares) we have converted the II a to II $\gamma$ using an average Balmer decrement from several solar and stellar ftares: $I I \gamma=1 / 3 M a$. As this ratio will vary' somewhat, according to the temperature and pressure in the regina of formation of the Balner lincs, (sec: Drike and Ulrich, 1980), we have preferred to use a mean value desermined cmpirically from olsservation.

\section{Observations}

A coordinated observation campaign by Kabler et a!. (1982) on YZ CMI was one of the first to obtain good simultaneous optical and X-ray coverage of a stellar farc. Gromul-based telescopes provided fluxes for the $H_{i}, 3$ and $H \gamma$ Balmer lines and the Imaging Proportional Counter (IPC) of EINSTEIN provided soft X-ray fluxes over the 0.2-1 KeV range. Integrated fluxes, above the normal 'equict' level for the entire flare event, were determined. As, to our knowledge, this was the only flare seen by EINSTEIN which had simultaneous Balner line coverage, whereas a mumber were observed by EXOSAT, we bave cunverted the EINSTEIN IPC 
flux $(0.2-4 \mathrm{~K} \cdot \mathrm{V})$ to the equivaleut EXOSAT LE flux $(0.04-2 \mathrm{KeV})$ by multiplying by a factor of three. This value was drtermined by Pallavicini et al. $(1980,1088)$ from observations of dMe stars by foth sutellites. The use of this factor implicitly assumes that the spectrum of the emission from flares is the same as that for the (quiet stars; an assumption which appears to be reasonably well justified by the determination of temperatures of flare plasmats by the EXOSAT ME experiment, (see Doyle et al. 1988a and Haisch et al. 1987) and the lack of any correlation of the ratio of IPC/EXOSAT flux with the derived temperature of the enitting plasma, (see figures 1 and 2 of Pallavicini ct al. 1986).

Three other sets of data, all of which involve EXOSAT observations of stellar flares, have been analysed. These include the flares on YZ CMi and Gl 644AB observed by Doyle et al. (1988a,b) and the small flares recorded on UV Ceti by Butler et al. (1985, 1980). The flare on YZ CMi was unusual in that there was no strikingly obvious event in LE X-rays simultaneous with the 1.3 magnitude optical event. Simultaneous spectroscopy showed a two-fold increase in the $H_{\gamma}$ flux during the U-band flare. The existence, or nonexistence, of an X-ray event at this tine is problematical, as although Doyle et al. concluded that there was no simultaneous event, there was a clear increase in LE X-ray flux about eleven minutes after the peak of tha U-band flare. The X-ray enlancenent at this time reached a peak of twice the normal level and lasted for ahout ten minutes, (see figure 1). Although the delay between the rise in X-rays and the rise in the U-band is rather longer than is normally cncountered in solar flares, we are incliued to believe that this was in fact the X-ray event associated with the promincent U-band flare at $19.55 \mathrm{UT}$, and that the apparent delay in the $X$-rity enhancencut is due, in part at least, to the spiky nature of the optical event. Assuming that this is indeed the: case the integrated $I / \gamma$ and LE soft X-ray fluxes for this event were $3 \times 10^{29}$ ergs and $5.5 \times 10^{30}$ ergs respectively.

Several small flares were seen on the protutype UV Ceti during a coordinated campaign in 1985 by Butler ct al. (1986) involving EXOSAT and ground-based telescopes. These events, which followed one another in the spatce of three hours, were referred to as micro-flares by the authors, not becanse of any sinilarity to the hard X-ray bursts seen by Lin et al. (1984), and also termed micro-flares, hut because they were small by comparison with the stellar flares commonly observed. In fact Butler et al (1986) and Collura et al (1988) have pointed out that these llares are roughly equivalent in size and duration to compact solar flares. For this study only the four largest of the small flares seen on UV Ceti, those termed A,B,C and D in figure 2, have been included. As with all the flares included here a correction has been made for the background 'quict' cmission.

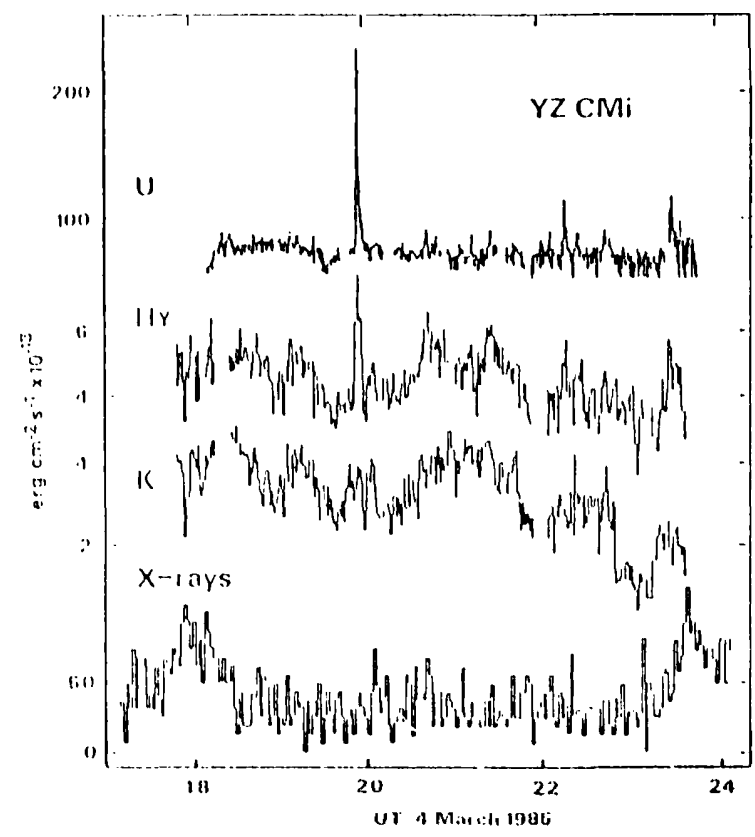

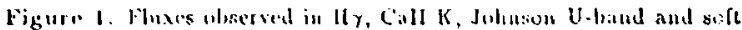

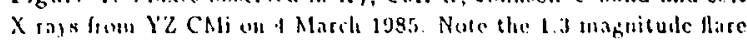

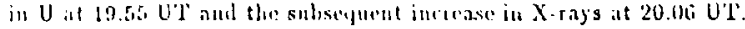

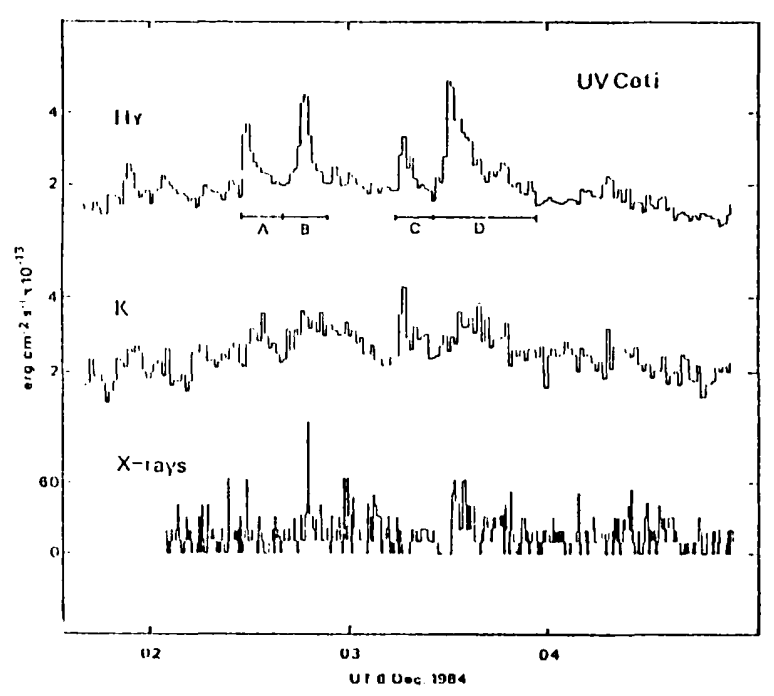

Figure 2. Fluxes obscried in Il $\gamma$, Call $K$ aud coll $X$-rays fiom UV Ceti on $G$ December 198. J Jie: four sonall flares $A, B, C$ and $D$ are iadiratol. 
One further stellar flare was observed by EXOSAT in soft X-rays and in Balmer emission $(H \alpha)$, on the bright dMe star Gl 644AB, by Doyle et al. (1988a). This is by far the most energetic of the flares discussed here with integrated fluxes an order of magnitude greater than the fluxes from the YZ CMi fare by Kaller et al. (1982).

It may seem surprising but there are almost no suitable observations of solar flares which can be compared to the above observations of stellar flares. Most of the published SMM data refer to the hard X-ray region and even for this there are very few simultaneous Balmer line measurements. The soft X-ray region (0.04-4 KeV) in which stellar flares have been observed by EINSTEIN and EXOSAT does not seem to have been popular with solar observers since the early rocket experiments. Indeed the lack of a single spectrum of this region for a solar flare makes it very difficult to scale the flux ul $\triangle M M$ instruments to the wavelength range of EINSTEIN and EXOSAT. The problern is compounded by the large number of emission lines that occur in the EUV soft X-ray region. However, for one solar flare, observed by Acton et al. (1982) in $H \alpha$ and the $1.85 \mathrm{~A}$ line of FeXXV, an estimate of the total soft X-ray flux, over the EXOSAT range (0.04-2 KeV) has been made by Doyle et al. (1088a) based on Acton's published emission measure curve and the emissivity function of Mewe et al. (1985). The integrated $H \alpha$ flux has been determined from Acton's time profile and converted to the $\mathrm{H}_{\gamma}$ flux using the same factor of $1 / 3$ used for the flare on Gl $644 \mathrm{AB}$.

The data for all the above flares are given in table 1. It is noticeable that the total integrated energies for the flares listed range over four orders of magnitude in both $H \gamma$ and soft $\mathrm{X}$-ray flux. Plotted in figure 3 we see an excellent correlation between the integrated $H \gamma$ and soft $\mathrm{X}$-ray flux for all the flares observed. The

Talsle 1. Integraterl II $\gamma$ and soft X-ray fluxes for flares on dMe stars and llic Sun

\begin{tabular}{|c|c|c|c|c|}
\hline Star & $\begin{array}{c}\text { distance } \\
\text { pursec }\end{array}$ & $\begin{array}{c}\mathrm{I}(\mathrm{II} \gamma) \\
\times 10^{28} \mathrm{erg}\end{array}$ & $\begin{array}{c}\mathrm{Lx}(0.04-2 \mathrm{KeV}) \\
\times 10^{28} \mathrm{erg}\end{array}$ & detector \\
\hline UV Ceti & 2.6 & 3.3 & 58 & EXOSAT \\
& & 4.9 & 260 & \\
& & 2.4 & 63 & \\
& & 12.0 & 560 & \\
YZ CMi & 6.1 & 320 & 10800 & EINSTEIN \\
YZ CMi & 0.1 & 30 & 550 & EXOSAT \\
Gl G4AAB & 6.4 & 2300 & 120000 & EXOSAT \\
Sun & $1 \mathrm{AU}$ & 0.2 & 12 & SMM \\
\hline
\end{tabular}

quality of the correlation is particularly striking when it is realised that the slope of the line drawn through the points has not been fitted but has slope unity in the $\log \log$ plot. This implies that there is a direct proportionality between $H \gamma$ and soft X-ray flux, $\mathrm{Lx}=31.6 \mathrm{~L}(H \gamma)$, for flares separated over four orders of magnitude in energy which includes flares on the Sun and nearby dMe stars. The tightness of the correlation is even more remarkable since observations of the fluxes were made by three different satellites and five different ground-based telescopes. This may well be the first time solar flares have been shown to obey a common relationship with flares on dMe stars.

\section{Interpretation}

The existence of a direct proportionality between the $H \gamma$ and X-ray flux for all flares for which we have the relevant data is unexpected for the following reasons.

1. Balmer emission arises from a plasma with a temperature of the order of $10^{4} \mathrm{~K}$, whereas the X-ray emission originates in a plasma with a temperature between $10^{6}-10^{7} \mathrm{~K}$. It is not obvious that there should be any correspondence in the emission between the two plasmas.

2. Time profiles of the Balmer emission show an appreciable impulsive phase component (see Kahler et al. 1982), whereas the soft $X$-rays are predominantly believed to arise from the cooling plasma in the more gradual and later thermal phase. 
3. The lower Bither lines ( $H \alpha, H_{/}, H_{\gamma}$ etc) are optically thick at chromosjueric deisities and therefore the: total integrated fluxes would be expected to be dependent on the projected area of the hydrogen ensitting plasma, rather thisn the volume, as in the case of the X-ray enitting plasma.

The unodels connuonly proposed for flares, and confirmed to some extent by spatially resolved obsurvation of soliar Harres, snggest that the Balner entission originates primarily in the footpoints of nangnetic loops where the deeper chemosphere and photosphere is heated to a temperature close to $10^{-4} K$ by the energetic particles which are leaned contes the surface by the magutic field. The soft X-rays, on the other hand, are supposed (1) orifginate: from the hot matrial evaporated from the foot points, which subsequently fills the magnetic lonps, and cools gradually by radiation. A simple model by de Jager $(1985,1987)$ pictures the region in the

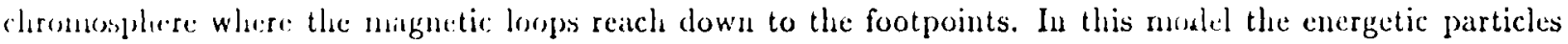
burn disw into the chromosphere leaving a hole from which the material has been evaporated. It is suggested that tha Baldurer enission arises from the lower surface of the hole, where the temperature is $\approx 10^{4} K^{5}$, and $N_{t} \approx 10^{13} \mathrm{~cm}^{-3}$. If the Balmer emission were to be optically thick at this density then it would be expected 16 sale: with the surface areat of the buttom of the hole whereas the X-ray emission would be proportional 16. He anumut of mattrial evaporated. Thus, one would naivcly expect, for stars with a similar atmospheric

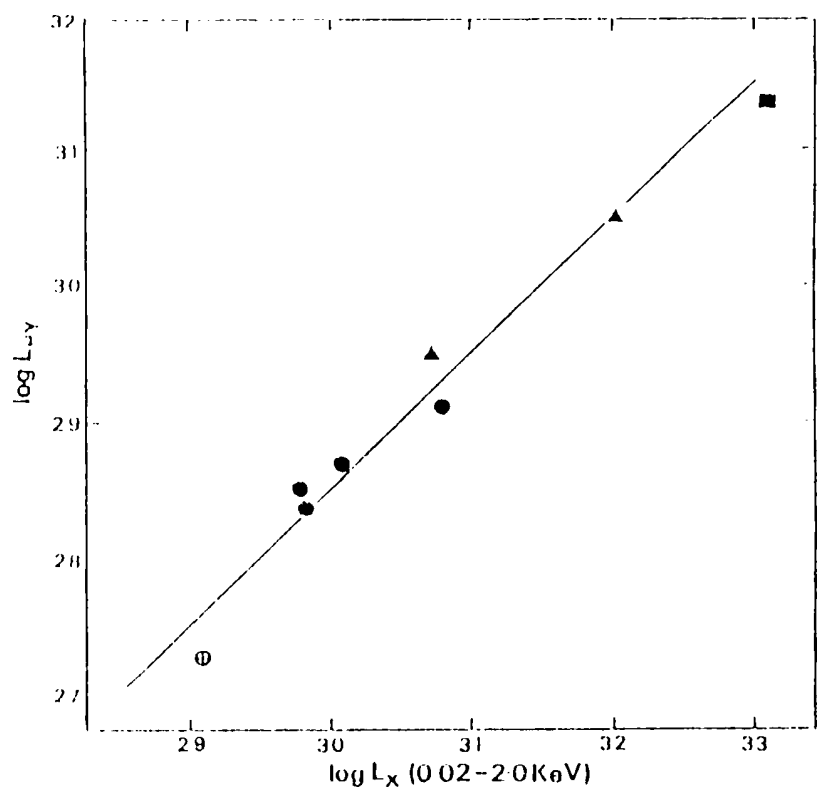

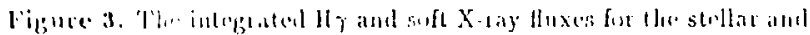

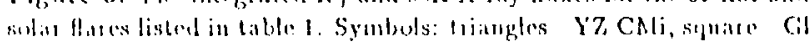

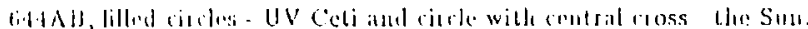

stricture, that a direct proportionality between Balmer and soft X-ray emission would imply that the ratio of the volume of the luble to its surface area, would be constant; i.e. that the well has a constant depth for flares wn all dMe stars and the Sum. Sinilarly, if the Balmer emission were to be optically thin, the proportionality hetweru Baluner and soft $X$-ray flux would ingly a constint ratio of the number of particles in the Balmer 'nvilling region to the number originally contained in the well. Other flare models by McCalse (1973) and l'ricst (1981) suggest that the Balmur emission arises from loops which lic below the soft X-ray enitting loops hut are clositly silated to them. The proportionality between the soft $\mathrm{X}$-ray and Balmer emission confirms this: closic assuchation but might inpose a tight constraint on their geometry.

A simpler explanation would be that the Bahmer Hux is a direct consequence of the soft X-ray flux and that the excitation of the liysogen atoms is primarily due to the soft $\mathrm{X}$-ray radiation rather than heating hy energetic particles. In this context it should be remembered that Zirin (1978) noted the simultaneous

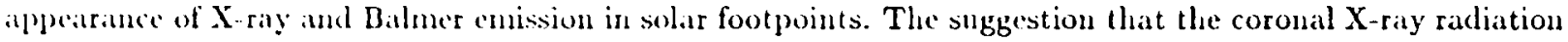
is an inpurtant contributor to the heating of the upper chromosphere in dMe stars was made by Cram (1982). If this mollel were to apply to the heating of the lower chromosphere by soft $X$-rays emitted by flares, and we insume that the downward directed $X$-ray thux is half the total Hux emitted, (where the total flux is emitted 
over $4 \pi$ steradian), then the total encrgy emitted in all hydrogen lincs should be less than half that emitted in soft X-rays. From Balmer decrement ratios observed in stellar flares by Doyle et al. (1988b) and Phillips et al. (1958) and similar ratios for quiet dMe stars by Gershiserg (1973) we would estimate the total Balmer flux to be $\approx 11 L(H \gamma)$. The Lyman flux is more difficult to estimate but from the computations of Drake and Ulrich (1980) the Lyman/Balmer ratio may be expected, for $T_{e} \approx 10^{4}$, to vary from unity for $\log N_{e}>13$ to ten for $\log N_{e}<10$. Thus, at the very least, our total estimited Balmer + Lyman Hux would be $\approx 22 L(H \gamma)$, i.e. about $2 / 3$ of the total soft $X$-ray flux. If $t^{\prime}$ ' amount of Lyman flux exceeded the Balmer flux by a factor greater than 1 , then the discrepancy is even greater, and the soft $X$-ray flux alone would be quite insufficient to explain the total hydrogen emission, let alone the emission in the other chromospheric lines. However, we should also include the flux in the EUV region, $\lambda \lambda 270-1216 \mathrm{~A}$ which lies outside the EXOSAT LE range, and will contribute to the excitation of the hydrogen atoms. In the absence of any definitive observations we estimate its energy as roughly $50 \%$ of the EXOSAT LE soft X-ray flux. Thus it appears that the observed hydrogen emission flux, $L(H) \approx 22 L(I I \gamma)$, is approximately equal to the total downward directed soft X-ray and EUV flux, $L_{D}(X+E U V)=1 / 2(32 L(H \gamma)+16 L(H \gamma)) \approx 24 L\left(H_{\gamma}\right)$. We conclude, therefore, that if the soft $X$-ray and EUV radiation were to be directly responsible for the hydrogen emission, a very efficient mechanism for conversion of the avalable radiation into hydrogen excitation would be required. Whatever the explanation, if the proportionality between soft $\mathrm{X}$-rays and $H_{\gamma}$ liolds, it means that one cin predict the soft $X$-ray flux, which is a sizeable fraction of the total radiated energy from a flare (approx. 30\%), from the measurement of the flux of just one Balner liue.

\section{References}

Acton,L.W., Canfiek, R.C., Gunkler,T.A., Iludson,H.S., Kiplinger,A.L. and Liebacher,J.W.: 1082, Astrophys. J. $269,409$.

Butler,C.J. and Rodono,M.: 1985, Irish Astr. J. 17, 191.

Butler,C.J., Rodono,M. Foing,B.H. and Haisch, B.M.: 1986, Nature 321, 679.

Collura, A., Pasquini,L. and Schmitt,J.H.M.M.: 1988, Astron. and Asrophys. (in press)

Cram, L.E.: 1982, Astrophys. J. 259, 768.

de Jager, C.: 1985, Solar Phys. 98, 267.

de Jager, C.: 1987, ibid 110, 337.

Duyle,J.G., Butler,C.J., Callanan,P.J., Tagliaferri,G., de la Reza,R., White,N.E., Torres,C.A. and Quast,G.: 1988a, Astron. and Astrophys. 191, 79.

Doyle,J.G., Butler,C.J., Byrne,P.B. and Van den Oord,G.H.J.: 1988b, Astron. and Astrophys. 199, 229.

Drake, S.A. and Ulrich, R.K.: 1980, Astrophys. J. Suppl. \{2, 351.

Gershberg, R.E.: 1973, Soviet Astrophys. 6, 02.

IIaisch,B.M., Butler,C.J., Doyle,J.G. and Rodono,M.: 1987, Astron. and Astrophys. 181, 96.

Kaller,S. et al.: 1982, Astrophys. J. 252, 299.

Lin,R.P. Schwartz,R.A., Kane,S.R., Pelling,R.M. and Ilurley,K.C.: 1984, Astrophys. J. 928, 121.

McCabe, M.K.: 1973, Solar Phys. 30, 439.

Mewe,R., Gronenschild,E.II.B.M. and Van den Oord,G.H.J.: 1985, Astron. and Astrophys. Suppl. 62, 197.

Pallavicini,R., Monsignor-Fossi,B.C. and Landini,M.: 1980, 'Cool Stary Stellar Systems and the Sun' proc. 4th Cambridge Cool Star Workshop, Santa Fe, 1985, Springer-Verlag, p 212.

Pallavicini,R., Monsignor-Fossi,B.C., Landini,M. and Schnitt,J.II.M.M.: 1988, Astron. and Astrophys. 191, 109.

Pricst, E.R.: 1981, 'Solar Flare Magnetohydrodynamics' Gorlon and Breach (pubs).

Zirin,H.: 1978, Solar Phys. 58, 25.

Zirin,II., Leggett,M. and Patterson,A.: 1082, Solar Phys. 70, 382. 